\title{
Betatrophin knockdown induces beiging and mitochondria biogenesis of white adipocytes
}

\author{
Zhe-Zhen Liao*, Xiao-Yan Qi*, Ya-Di Wang, Jiao-Yang Li, Qian-Qian Gu, Can Hu, Yin Hu, Heng Sun, Li Ran, Jing Yang, \\ Jiang-Hua Liu and Xin-Hua Xiao \\ Department of Metabolism and Endocrinology, The First Affiliated Hospital of University of South China, Hengyang, Hunan Province, China \\ Correspondence should be addressed to X-H Xiao: xinhua0102@163.com
}

*(Z-Z Liao and X-Y Qi contributed equally to this work)

\begin{abstract}
Remodeling of energy-storing white fat into energy-consuming beige fat has led to a promising new approach to alleviate adiposity. Several studies have shown adipokines can induce white adipose tissue (WAT) beiging through autocrine or paracrine actions. Betatrophin, a novel adipokine, has been linked to energy expenditure and lipolysis but not clearly clarified. Here, we using high-fat diet-induced obesity to determine how betatrophin modulate beiging and adiposity. We found that betatrophin-knockdown mice displayed less white fat mass and decreased plasma TG and NEFA levels.

Consistently, inhibition of betatrophin leads to the phenotype change of adipocytes characterized by increased mitochondria contents, beige adipocytes and mitochondria biogenesis-specific markers both in vivo and in vitro. Of note, blocking AMP-activated protein kinase (AMPK) signaling pathway is able to abolish enhanced beige-like characteristics in betatrophin-knockdown adipocytes. Collectively, downregulation of betatrophin induces beiging in white adipocytes through activation of AMPK signaling pathway. These processes suggest betatrophin as a latent therapeutic target for obesity.
\end{abstract}

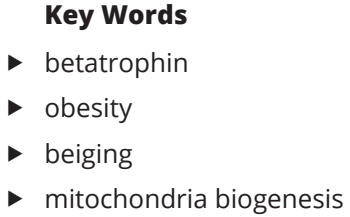

Journal of Endocrinology (2020) 245, 93-100

\section{Introduction}

Obesity, characterized by excess fat accumulation, is a major risk factor for developing type 2 diabetes (T2D), cancer and cardiovascular disorders (Jenkins et al. 2018). When energy intake exceeds energy expenditure, the excess energy is stored as fat in white adipose tissue (WAT). However, recent studies indicated that white adipocytes can undergo 'beiging' process to generate heat. The cells involved in the 'beiging' process, are known as beige adipocytes, have similar molecular characteristics to brown adipocytes including abundant mitochondrial content and high uncouple protein 1 (UCP-1) expression (Ikeda et al. 2018). UCP-1 plays a role in mediating proton leak and induces uncoupled respiration. Through this process, beiging of WAT increases the utilization of nutrients and may contribute to whole-body energy homeostasis.

The factors involved in the determination of beige adipocytes fate are not fully understood. A number of adipokines such as FGF21 (Veniant et al. 2015), BMP4 (Hoffmann et al. 2017) and ZAG (Elattar et al. 2018) have been reported to promote adaptive thermogenesis upon cold exposure. Recently, betatrophin has been identified atypical member of the angiopoietin-like protein family as a regulator of triglyceride in plasma through its interaction with ANGPTL3 and its regulation of lipoprotein lipase activity (Zhang 2012, Izumi et al. 2018). It has been 
indicated that serum and adipose tissues of betatrophin are positively correlated with obesity and lipogenesis (Abu-Farha et al. 2016). More strikingly, a latest rodent study further demonstrated that betatrophin-deficient mice have decreased fat mass and a prominent increased energy expenditure (Gusarova et al. 2017, Banfi et al. 2018), implying that betatrophin may also act as an antithermogenesis adipokine. Surprisingly, previous reports argued that betatrophin expression is markedly upregulated in adipose tissues upon cold stimulus (Fu et al. 2013). Given the increased expression of betatrophin upon cold exposure would be expected to promote thermogenesis, which leads us to hypothesize whether betatrophin may be a beige-like adipokine like FGF21, BMP4, and so forth. (Qian et al. 2013). Apparently, the hypothesis is difficult to reconcile with the aforementioned literature. For this reason, these contradictory findings would question the role of betatrophin in WAT beiging. Hence, the objective of this study is to investigate the physiologic impact of betatrophin in WAT beiging by employing a loss-offunction approach in mice.

Here, we discovered that inhibition of betatrophin attenuated white fat mass and improve TG levels through stimulating beiging process and mitochondria biogenesis. The above process was mainly dependent on the activation of AMPK signaling pathway. These results demonstrated the role of betatrophin as a target to modulate mitochondrial function and beige remodeling in the WAT.

\section{Materials and methods}

\section{Animals and treatment}

Male C57BL/6J mice were purchased from the Model Animal Research Center of Nanjing University (Nanjing, China). Four-week-old C57BL/6J male mice were housed under standard laboratory conditions $\left(26^{\circ} \mathrm{C}, 12\right.$-h light/ dark cycle) with free access to food and drinking water. At the age of 8 weeks, mice weighing 24-28 g were divided into four distinct groups ( $n=8$ for each group) and one group was subjected to standard-fat diet (SFD): protein $20 \%$, fat $10 \%$ and carbohydrates $70 \%$ and the other three groups were subjected to high-fat diet (HFD): protein $20 \%$, fat $60 \%$ and carbohydrates $20 \%$. After 12 weeks, the HFD mice were respectively transducted with rAAV2-betatrophin RNAi $(n=8)$ or rAAV2-GFP $(n=8)$ once via tail vein injection $(5 \times 10 \times 10 \mathrm{~g})$ under HFD for another 4 weeks before killing. Body weight and food intake were measured weekly. Serum and adipose tissues were collected for further analysis. All procedures were reviewed and approved by the Animal Welfare Committee at the Institute of Experimental Medicine, University of South China.

\section{HE staining and immunohistochemical analysis}

Fresh s.c. white adipose tissue (sWAT) and epididymal white adipose tissue (eWAT) were fixed in 10\% formalin/PBS, and then embedded with paraffin. Sections were subjected to standard hematoxylin-eosin (H\&E) staining as previously described (Zhang et al. 2018). Quantitative image analysis was performed by computerized densitometry using the ImagePro Plus 7 program (Media Cybernetics, Silver Spring, MD, USA). For immunohistochemical analysis, cryosections were stained with anti-UCP1 antibody (Abcam) following standard procedures.

\section{Plasma non-esterified fatty acids (NEFAs) and TG measurement}

Blood samples were collected from 24-week-old mice, and the plasma was isolated by centrifugation $(300 \boldsymbol{g})$ for 10 min. Plasma TG and NEFA were determined with TG kit (SHENSUO UNF, China) and Wako HR Series NEFA-HR kit (Wako Pure Chemical Industries), respectively.

\section{Cell differentiation and transfection}

Mouse 3T3-L1 preadipocytes (Zenbio, Durham, NC, USA) were grown and differentiated as previously described (Zebisch et al. 2012). Differentiated adipocytes were transfected with rAAV2-betatrophin RNAi according to the manufacturer's instructions. Additionally, mature adipocytes were pre-transfected for $72 \mathrm{~h}$ with the rAAV2-RNAi and then incubated with AMPK inhibitors (compound C $20 \mu \mathrm{M}$ ) for $2 \mathrm{~h}$.

\section{Electron microscopic observation}

3T3-L1 adipocytes were washed with $0.1 \mathrm{M}$ phosphate buffer and fixed in $2.5 \%(\mathrm{w} / \mathrm{v})$ glutaraldehyde solution overnight at $4^{\circ} \mathrm{C}$. Then, cells were post-fixed in $1 \%(\mathrm{w} / \mathrm{v})$ osmium tetraoxide, dehydrated in ascending gradations of ethanol and embedded in fresh epoxy resin. Ultra-thin sections $(70-80 \mathrm{~nm})$ were cut and stained with uranyl citrate and lead citrate before being examined under a transmission electron microscope Philips CM-10. (c) 2020 Society for Endocrinology Published by Bioscientifica Ltd.
Printed in Great Britain 


\section{Immunofluorescence analysis}

3T3-L1 adipocytes were fixed and blocked with 1\% BSA in PBST for $1 \mathrm{~h}$. Cells were then incubated with antiUCP1 antibodies (Abcam) at $4^{\circ} \mathrm{C}$ overnight, followed by incubated with FITC-conjugated secondary antibody. DAPI (Invitrogen) was used to stain the nuclei of cells. Fluorescence images were captured with a Bio-Rad confocal microscope (MRC 1024; Bio-Rad).

\section{Mitotracker staining}

For WAT of mitochondria staining, deparaffinized slices of WAT from rAAV2-GFP and rAAV2-RNAi mice were incubated with 250 nM MitoTracker Red (Molecular Probes) for $1 \mathrm{~h}$ at room temperature. For staining mitochondria of 3T3-L1 cells, MitoTracker Red was directly added to growing media following the manufacturer's protocol and cells were incubated for $1 \mathrm{~h}$ at $37^{\circ} \mathrm{C}$, and then observed with a Bio-Rad confocal microscope (MRC 1024; Bio-Rad).

\section{Western blotting}

Fat tissues were collected and immediately frozen in liquid nitrogen. Proteins were extracted with RIPA buffer (Cell Signaling Technology) supplemented with complete protease inhibitor cocktail (Roche). Then, protein samples were subjected to SDS-PAGE and transferred to PVDF membranes. After blocking with 5\% skim milk, membranes were incubated with the appropriate primary antibodies. Primary antibodies in this study are listed in Supplementary Table 1 (see section on supplementary materials given at the end of this article). After washing three times in PBST, blots were incubated with the appropriate horseradish peroxidase-conjugated secondary antibodies at $37^{\circ} \mathrm{C}$ for $1 \mathrm{~h}$. Western blots were developed using ECL (Beyotime Institute of Biotechnology, China) and quantified by scanning densitometry.

\section{Quantitative real-time PCR (qRT-PCR)}

Total RNAs from 3T3-L1 cells were extracted using TRIzol Reagent (Invitrogen) according to the manufacturer's instructions. cDNA was prepared from $500 \mathrm{ng}$ of RNA using the PrimeScript RT Master Mix (TaKaRa) diluted to a final volume of $40 \mu \mathrm{L}$. Then, $1 \mu \mathrm{L}$ of diluted cDNA was used in a $20 \mu \mathrm{L}$ PCR reaction with SYBR Green Master Mix (Applied Biosystems). The PCR reactions were run in triplicate for each sample and quantified using the ABI Prism 7300 Sequence Detection System (Applied Biosystems). Gene expression levels were calculated by normalizing to
$18 \mathrm{~S}$ RNA by the $2^{-\Delta \Delta \mathrm{Ct}}$. All the primers were synthesized by Sangon Biotech (Shanghai, China). The primer pairs used in this study are listed in Supplementary Table 2.

\section{Statistical analysis}

All values are expressed as means \pm s.D. of no less than three separate experiments. Statistical significance was determined using the $t$ test between the two groups by the Statistical Package of Social Science (SPSS, version 17.0) program. Statistical significance was set $a$ priori at $P<0.05$.

\section{Results}

\section{Betatrophin knockdown improves adiposity and plasma TG levels in HFD-induced obese mice}

In order to elucidate the role of betatrophin in obesity, HFD-fed mice were transducted efficiently with rAAV2betatrophin RNAi or rAAV2-GFP via the tail vein. As expected, the protein and mRNA expression of betatrophin were dramatically decreased in the eWAT and sWAT (Supplementary Fig. 1A, B and C). Betatrophinknockdown mice showed no alteration in the final body weights (Fig. 1A) and food intake (Fig. 1B). We still observed that the ratio of white fat mass to body weights was significantly decreased in the betatrophinknockdown mice (Fig. 1C and D). Furthermore, inhibition of betatrophin induced the appearance of beige-like cells specifically within the WAT, which was confirmed by increased emergence of multilocular adipocytes and decreased adipocytes diameter (Fig. 1E and F). Additionally, the plasma TG and NEFA levels were dramatically decreased in betatrophin-knockdown mice (Fig. 1G and $\mathrm{H}$ ). Together, these results suggest that downregulation of betatrophin was able to alleviate adiposity, which might be attributed to the increased beiging potential of WAT.

\section{Betatrophin knockdown produces a beige-like phenotype in WAT}

To test the impact of betatrophin on the induction of WAT beiging, we examined one set of genes involved in beige adipocytes formation. As show in Fig. 2A and B, inhibition of betatrophin upregulated protein levels of UCP-1, PRDM16 and Cidea in both sWAT and eWAT. The beiging phenotype of adipocytes was further confirmed by immunohistochemical detection of UCP-1 (Fig. 2F). Given that mitochondria biogenesis is the key process for beiging, we compared the mitochondrial contents 
A

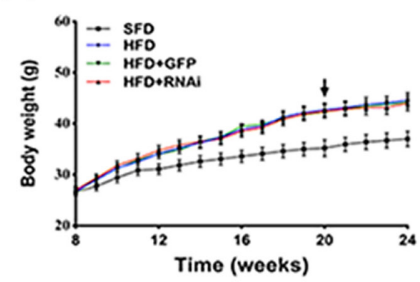

$E$

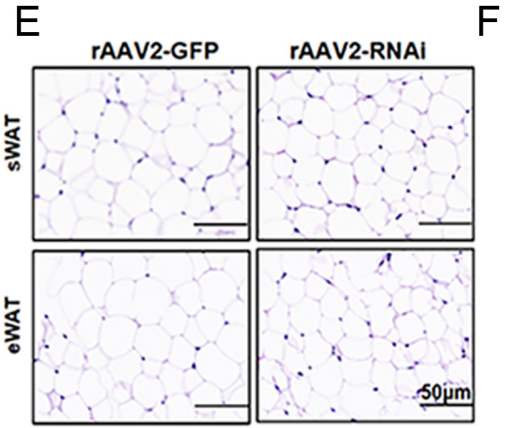

B

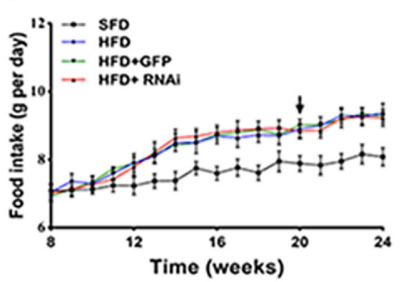

F

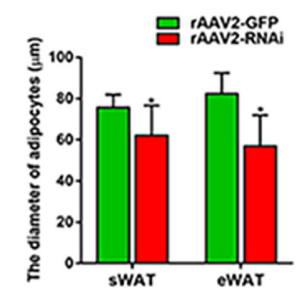

C

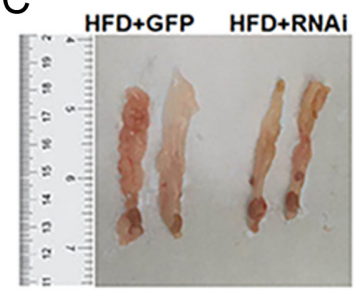

D

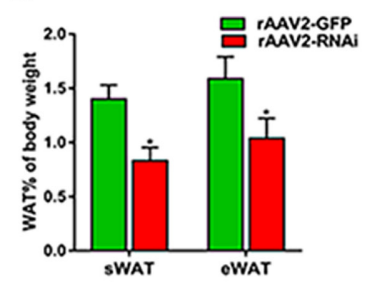

G

$\mathrm{H}$
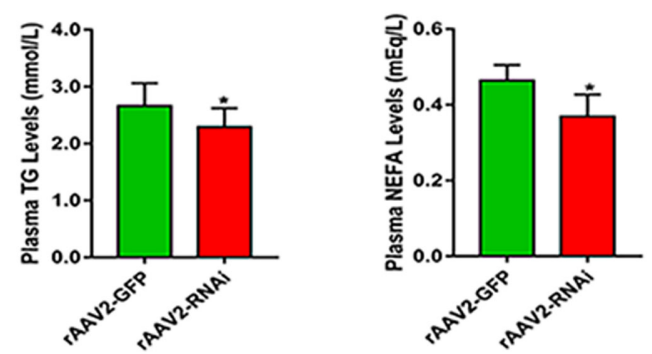

Figure 1

Inhibition of betatrophin decreased white fat contents in HFD-induced obese mice. Eight-week-old mice were fed with HFD for 12 weeks, and then received injections of rAAV2-RNAi or rAAV2-GFP through the tail vein over one month, $n=8$ per group. (A) Body weight and (B) food intake curves of rAAV2-GFP mice and rAAV2-RNAi mice fed with HFD. (C) Representative appearance of eWAT. (D) Ratio of sWAT and eWAT weight to body weight. (E) Representative H\&E staining of sWAT and eWAT. Scale bars $=50 \mu \mathrm{m}$. (F) The diameter of adipocytes in sWAT and eWAT. (G and H) Plasma TG and NEFA levels of rAAV2-GFP group and rAAV2-RNAi group $(n=8)$. Data are shown as mean \pm s.D., $n=8$. * $P<0.05, * \star P<0.01$, versus the rAAV2-GFP group.

between betatrophin-knockdown and control mice. As shown in Fig. 2E, betatrophin-knockdown mice have more mitochondria in WAT compared to control mice. Correspondingly, downregulation of betatrophin induced the protein expression of mitochondria biogenesis-related genes as well, such as PGC- $1 \alpha$, NRF-1 in WAT (Fig. 2C and $\mathrm{D})$. These results indicated that downregulation of betatrophin could induce a remodeling of white adipocytes via stimulating mitochondria biogenesis and beiging markers in WAT of obese mice.

\section{Betatrophin knockdown induces beiging and mitochondria biogensis in 3T3-L1 adipocytes}

Next, we wonder whether silencing betatrophin leads to beige fat remodeling in vitro. We firstly evaluated the knockdown efficiency of rAAV2-betatrophin RNAi in 3T3-L1 adipocytes. As expected, the mRNA and protein expression of betatrophin were significantly suppressed upon virus transfection (Supplementary Fig. 1D and E). In parallel with animal studies, increased expression of UCP-1 and other thermogenic genes were observed in adipocytes from betatrophin-silenced adipocytes (Fig. 3A and C). Moreover, immunofluorescence staining also showed a higher level of UCP-1 expression upon betatrophin inhibition (Fig. 3D). Additionally, we investigated the effect of betatrophin on mitochondria biogenesis in
3T3-L1 cells. A larger number of mitochondria was observed in mature adipocytes infected with rAAV-betatrophin RNAi (Fig. 3E and F), indicating that the mitochondrial abundance was enhanced upon betatrophin inhibition. In line with the observed in vivo, mitochondria biogenesismarkers, PGC-1 $\alpha$, NRF-1, NRF-2, and mtTFA remained upregulated in betatrophin-silenced 3T3-L1 adipocytes (Fig. 3B and C). In general, silencing betatrophin drove beiging process along with mitochondrial biogenesis in 3T3-L1 adipocytes.

\section{Betatrophin knockdown induces white adipocytes beiging via AMPK signaling pathway}

Since it has been reported that AMPK is a master switch regulating beiging process and energy homeostasis, we tested whether AMPK expression was altered when betatrophin was inhibited. Interestingly, increased phosphorylated level of AMPK and ACC was observed in WAT from betatrophin-knockdown mice (Fig. 4B). This phenotype is recapitulated in matured 3T3-L1 adipocytes (Fig. 4A). To further if the enhanced AMPK signaling induced by betatrophin inhibition is responsible for the beige-like phenotype, we blocked AMPK activity by compound $\mathrm{C}$ in betatrophin-silenced adipocytes. Of note, compound $\mathrm{C}$ indeed remarkably abolished the upregulation of beiging marker (UCP-1, PRDM16, 
A

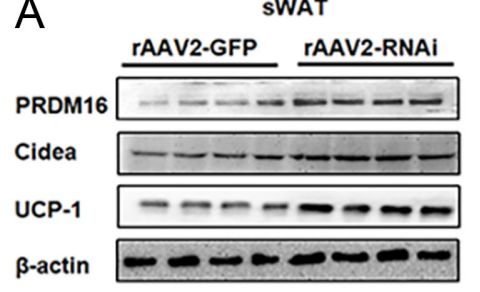

C

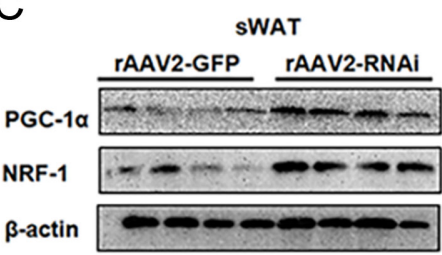

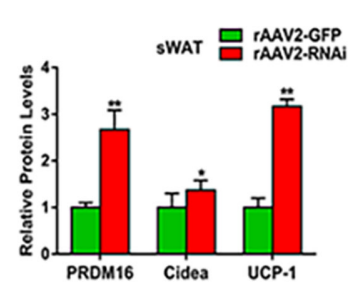

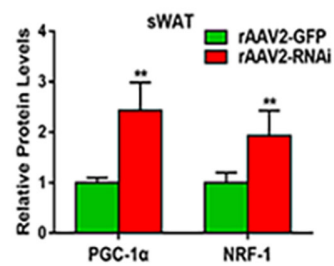

B eWAT
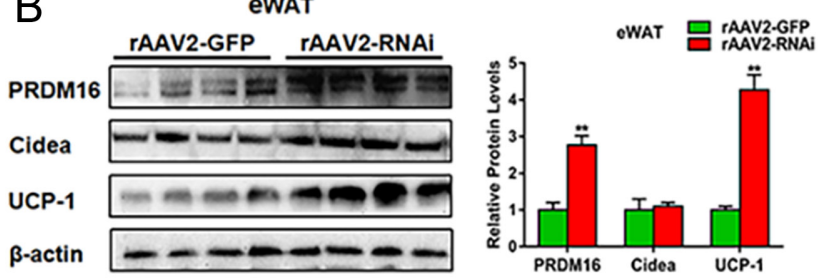

D
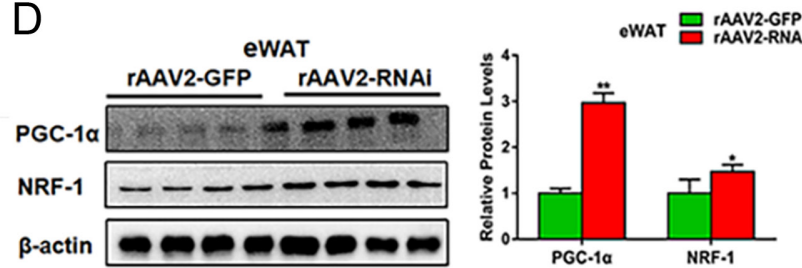

\section{E}
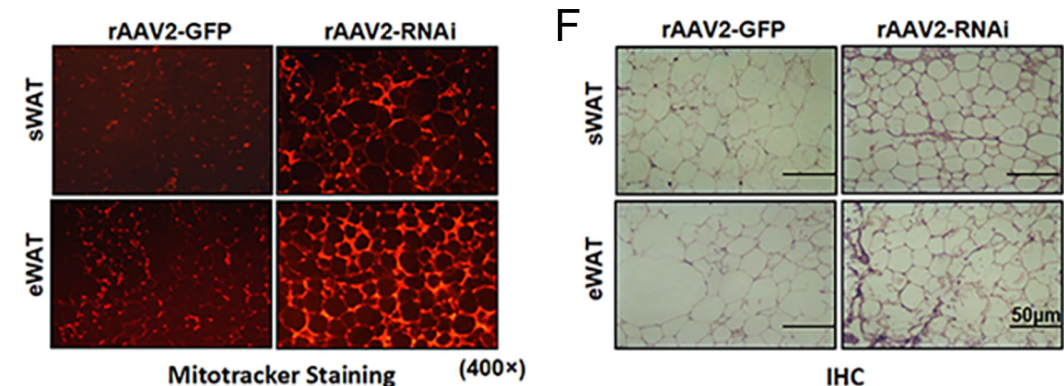

Figure 2

Inhibition of betatrophin induces beige-like phenotype and enhances the mitochondrial function in WAT of obese mice. (A and B) Quantification and representative Western blots of beige fat-related genes (PRDM16, Cidea, UCP-1) protein levels in sWAT and eWAT of control $(n=8)$ and rAAV2-RNAi mice $(n=8)$. (C and D) Quantification and representative Western blots of mitochondria biogenesis-related gene (PGC1- $\alpha$ and NRF-1) protein levels in sWAT and eWAT of control $(n=8)$ and rAAV2-RNAi mice $(n=8)$. (E) Mitochondrial staining of sWAT and eWAT in rAAV2-GFP mice and rAAV2-RNAi mice. Original magnification, 400×. (F) UCP-1 immunostaining in sWAT and eWAT from rAAV2-GFP mice and rAAV2-RNAi mice. Scale bars: $50 \mu \mathrm{m}$. Data are shown as mean \pm S.D., $n=8 . * P<0.05, * * P<0.01$, versus the rAAV2-GFP group.

Cidea, p-AMPK, p-ACC) and mitochondria biogenesis markers (PGC-1 $\alpha$, NRF-1) upon betatrophin inhibition (Fig. 4C, D and E). In addition to the AMPK signaling pathway, we detected the acitivtiy of cAMP-dependent protein kinase A (PKA) signaling pathway, which can also induce white fat beiging (Zhang et al. 2018). As shown in Supplementary Fig. 2, inhibiting betatrophin in adipocytes had no impact on the protein level of p-CREB and p-PKA. These results suggested that betatrophin could suppress the expression of p-AMPK at protein level. In this mode, betatrophin negatively modulates beiging and mitochondria biogenesis program that leads to beige-towhite phenotype switching (Fig. 4F).

\section{Discussion}

Plasticizing WAT to acquire beige-like features is a promising approach against obesity and associated complications (Giordano et al. 2016). Betatrophin promotes the recruitment of thermogenic beige adipocytes within the WAT, highlighting a novel mechanism by which betatrophin antagonist or antibody may alleviate adiposity. Furthermore, our findings show that the activation of AMPK signaling by betatrophin inhibition served as a new mechanism to remodel white adipocytes.

The role of betatrophin in regulating energy metabolism was previously studied, where betatrophin deficiency has a favorable effect on energy expenditure (Gusarova et al. 2017, Banfi et al. 2018). Despite these initial clues, our work highlighted the modulation of betatrophin on WAT plasticity. Classical features indicating the conversion of white fat into beige adipose includes multilocular lipid droplets, an abundant amount of mitochondria, and increased expression of thermogenic proteins such as PGC1 $\alpha$, Cidea ,UCP-1 (Holmes 2017). Based on the features of beige fat cells, we characterized mice with a loss of function of betatrophin in WAT and performed 


\section{A}
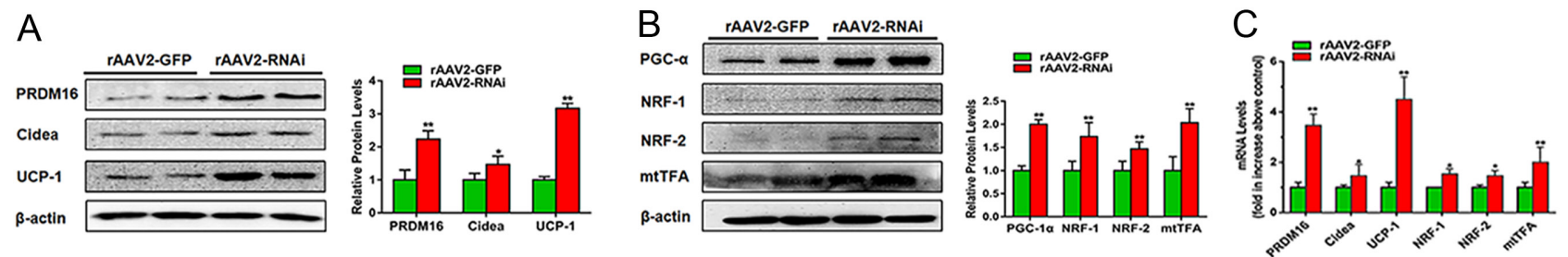

D
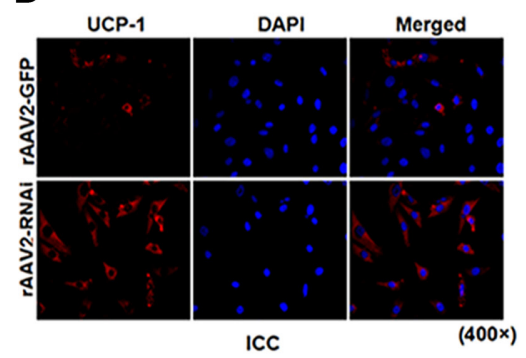

E

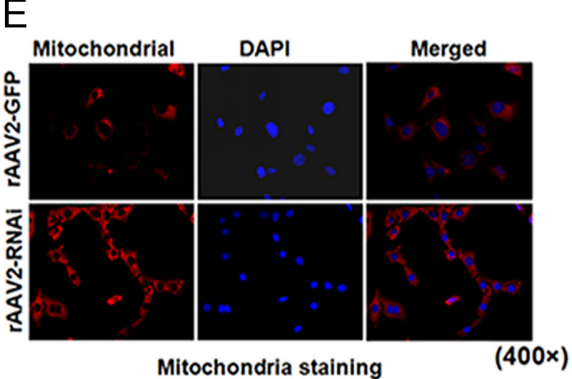

$\mathrm{F}$

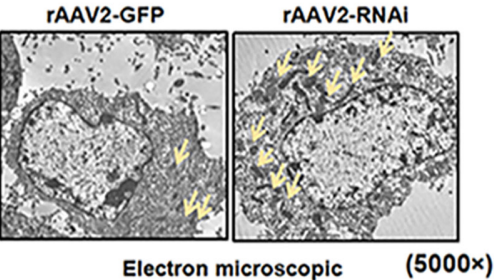

Figure 3

Inhibition of betatrophin promotes beiging in 3T3-L1 adipocytes. (A) Quantification and representative Western blots of UCP-1, PRDM16 and CIDEA in rAAV2-RNAi or rAAV2-GFP transfected adipocytes $(n=3)$. (B) Quantification and representative Western blots of protein expression levels of PGC-1 $\alpha$, NRF-1, NRF-2 and mtTFA in rAAV2-RNAi or rAAV2-GFP transfected adipocytes $(n=3)$. (C) Immunofluorescence staining of UCP-1 in rAAV2-RNAi- or rAAV2-GFPtransfected adipocytes. Original magnification, 400x. (D) qRT-PCR analysis of the beiging remodeling markers and mitcochondria biogenesis-related genes in 3T3-L1 adipocytes transfected with rAAV2-GFP or rAAV2-RNAi $(n=3)$. (E) Representative transmission electronic microscopic images from differentiated 3T3-L1 transfected with rAAV2-RNAi or rAAV2-GFP. Original magnification, 5000×. (F) MitoTracker Red staining of mitochondria in rAAV2-GFP and rAAV2-RNAi transfected 3T3-L1 cells. Original magnifcation, 400×. Data are expressed as means \pm S.D., $* P<0.05, * \star P<0.01$, versus the rAAV2-GFP group.

comprehensive studies in 3T3-L1 adipocytes. Both in vivo and in vitro models, we concluded that downregulation of betatrophin not only reduced the WAT content in obese mice but also transformed large adipocytes into small adipocytes filled with multilocular lipid droplets. Additionally, the abundance of mitochondria and beiging markers were significantly increased after suppressing betatrophin expression in WAT. Although our study demonstrated that betatrophin-knockdown mice had no alteration in final body weight, which contrasts with a prior report that betatrophin deficiency resulted in body weights loss (Banfi et al. 2018). The contradictory findings may be explained by the different strategies of genetic modification. Compared with virus-mediated partial betatrophin knockdown, complete knockout of the betatrophin gene is more efficient to reduce body weights. Hence, these results clearly demonstrated that specific inhibition of betatrophin is adequate to induce beige remodeling in WAT.

Next, we further investigated the relationship between betatrophin and AMPK signaling. AMPK is a sensor of intracellular energy that regulates the energy balance (Imran et al. 2018). The stimulative effects of AMPK on beiging of WAT have been extensively explored. Once activated, AMPK modulates WAT beiging through elevation of beige fat-selective proteins (PRDM16 and UCP-1) and concurrently promoting mitochondria biogenesis (Mottillo et al. 2016). AMPK activity was often increased under conditions, such as during exercise, fasting or in response to compounds that increase cAMP and PKA activity (like epinephrine), in which betatrophin expression was maximally suppressed in WAT (Tseng et al. 2014, Lee et al. 2015, Abu-Farha et al. 2016), suggesting a possible connection between betatrophin and AMPK. Surprisingly, a latest study revealed that betatrophin served as a negative regulator of AMPK signaling pathway, thereby suppressing hepatocellular carcinoma growth (Wang et al. 2018). In line with these reports, we demonstrated that betatrophin inhibited beiging of white adipocytes through blocking AMPK signaling pathway. Despite the current data providing an important hint that AMPK activation may be involved in betatrophin deletion-induced beiging in vitro, further investigations in adipose-specific AMPK mouse model are still needed to verify it. Additionally, it should also be noticed that loss of betatrophin may promote beige fat formation through other signaling pathways. Previous study demonstrated that betatrophin modulated lipid metabolism through enhancement of autophagy (Tseng et al. 2014). A recent report indicates inhibition of adipose mitochondrial autophagy favors maintenance of beige adipocyte ( $\mathrm{Lu}$ et al. 2018). Therefore, adipose-selective betatrophin knockout may also induce beige WAT formation through mitochondrial autophagy-related signaling pathways, which could be explored in the future. 
A
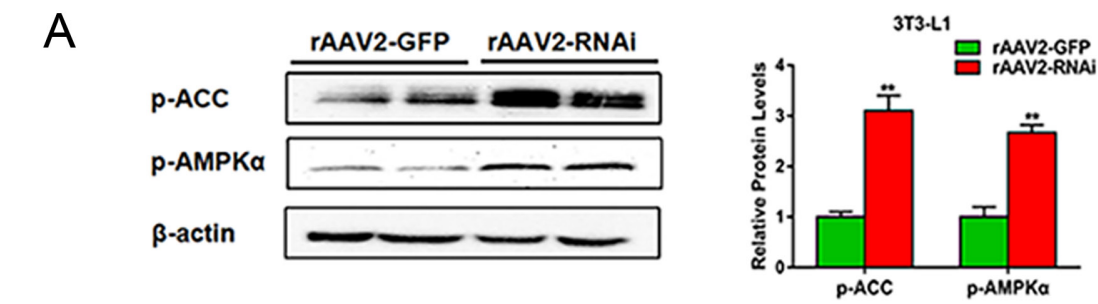

C

B
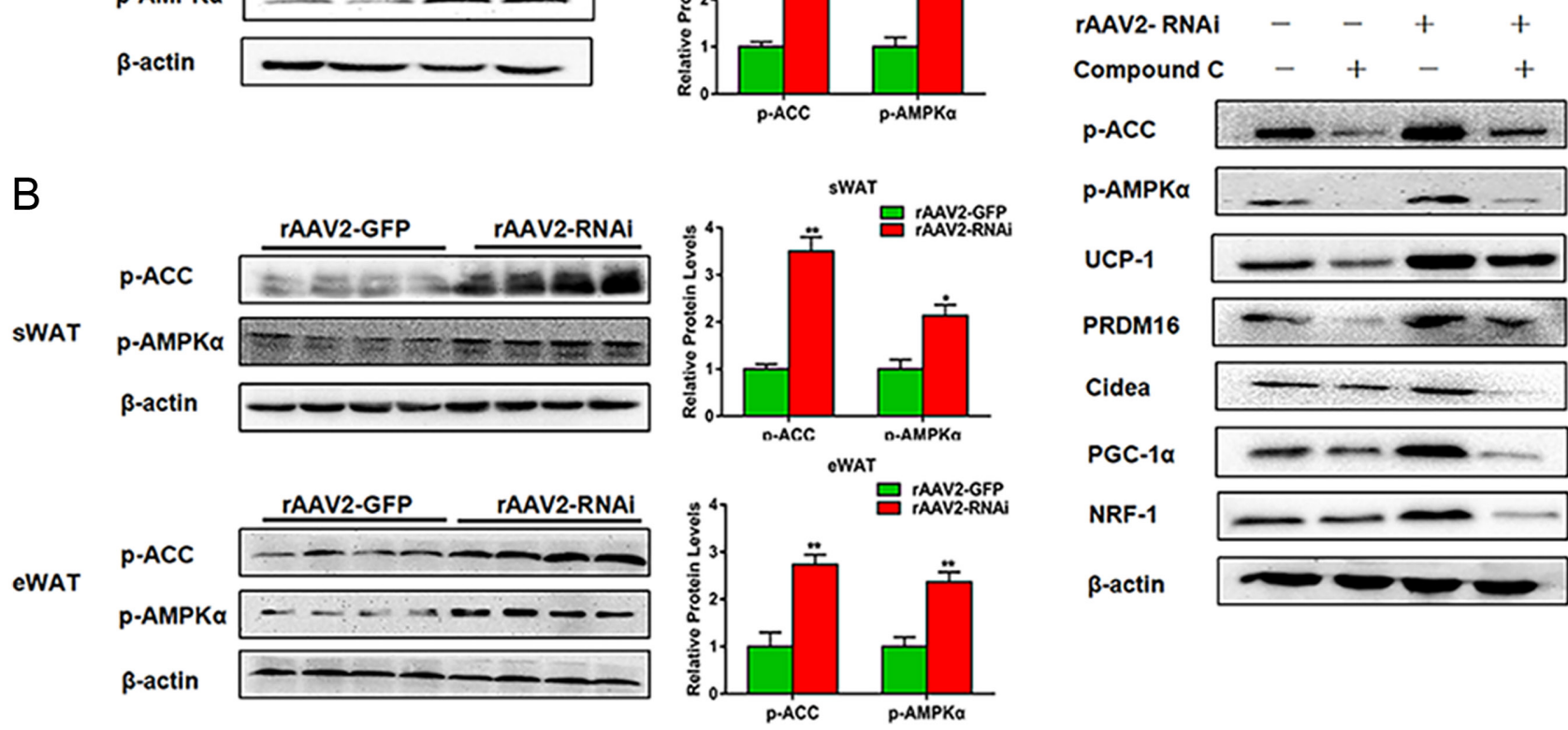

p-AMPKa

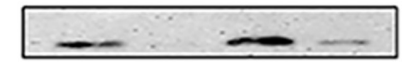

UCP-1

PRDM16

Cidea

PGC-1 $\alpha$

NRF-1

$\beta$-actin
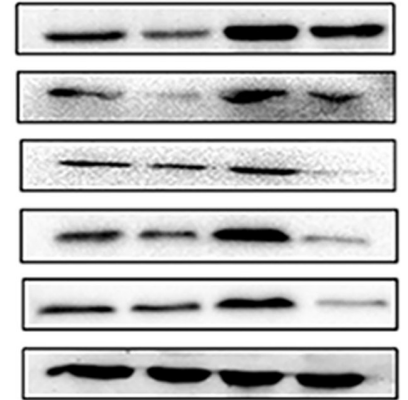

D

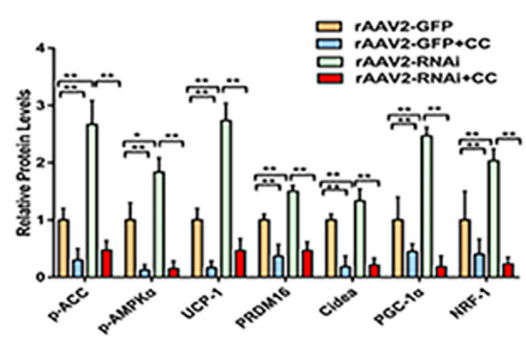

E

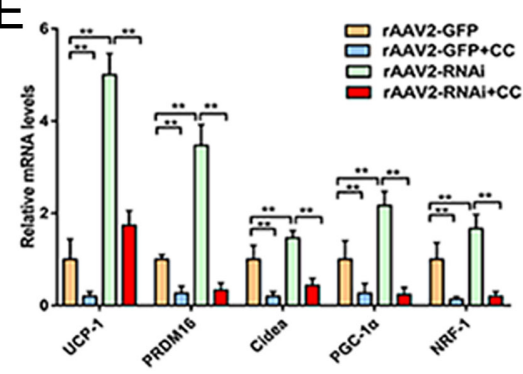

$\mathrm{F}$

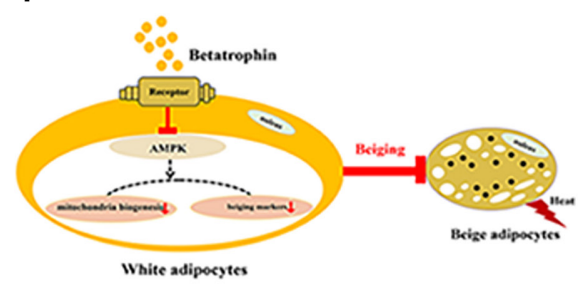

Figure 4

Inhibition of betatrophin enhances AMPK-dependent beiging in white adipocytes. (A) Quantification and representative Western blots of pAMPK, p-ACC in 3T3-L1 adipocytes transfected with rAAV2-GFP or rAAV2-RNAi $(n=3)$. (B) Quantification and representative Western blots of pAMPK, p-ACC in WAT of rAAV2-GFP mice and rAAV2-RNAi mice $(n=8)$. (C and D) Quantification and representative Western blots of pAMPK, p-ACC, UCP-1, PRDM16, Cidea, NRF-1, PGC1- $\alpha$ in 3T3-L1 adipocytes treated with compound C $(20 \mu \mathrm{M})$ for $2 \mathrm{~h}$. (E) qRT-PCR analysis of the beiging markers and mitcochondria biogenesis-related genes in 3T3-L1 adipocytes treated with compound C $(n=3)$. (F) Schematic diagram of the working model for the roles of betatrophin in inhibiting WAT beiging. Data are expressed as means \pm S.D., $* P<0.05, * \star P<0.01$.

It is well known that different fat compartments are associated with differential metabolic risk. Excessive visceral fat accumulation, also known as visceral obesity, is associated with poor prognosis, metabolic disturbances, and increased degree of pathology in several chronic diseases (Hwang et al. 2015). In our context, we find that inhibition of betatrophin facilitates stronger beiging capacity in epididymal fat compared to s.c. fat. The potential mechanisms may relate to the higher level of betatrophin in visceral fat and that obesity mainly leads to increasing expression of betatrophin in visceral fat rather than sWAT (von Loeffelholz et al. 2017). Taken together, we discovered the important role of betatrophin in the beiging of WAT and mitochondria homeostasis. Based on our findings, we propose that betatrophin in WAT could serve as a novel target to combat obesity and its accompanying diseases.

\section{Supplementary materials}

This is linked to the online version of the paper at https://doi.org/10.1530/ JOE-19-0447. 


\section{Declaration of interest}

The authors declare that there is no conflict of interest that could be perceived as prejudicing the impartiality of the research reported.

\section{Funding}

This work was supported by research grants from the National Natural Science Foundation of China (81870595), Major Scientific Research Projects of Hunan health and family planning commission (A2017011). The authors have no conflicts of interest with regard to the subjects of this paper.

\section{References}

Abu-Farha M, Sriraman D, Cherian P, AlKhairi I, Elkum N, Behbehani K \& Abubaker J 2016 Circulating ANGPTL8/Betatrophin is increased in obesity and reduced after exercise training. PLOS ONE 11 e0147367. (https://doi.org/10.1371/journal.pone.0147367)

Banfi S, Gusarova V, Gromada J, Cohen JC \& Hobbs HH 2018 Increased thermogenesis by a noncanonical pathway in ANGPTL3/8deficient mice. PNAS 115 E1249-E1258. (https://doi.org/10.1073/ pnas.1717420115)

Elattar S, Dimri M \& Satyanarayana A 2018 The tumor secretory factor ZAG promotes white adipose tissue browning and energy wasting. FASEB Journal 32 4727-4743. (https://doi.org/10.1096/fj.201701465RR)

Fu Z, Yao F, Abou-Samra AB \& Zhang R 2013 Lipasin, thermoregulated in brown fat, is a novel but atypical member of the angiopoietin-like protein family. Biochemical and Biophysical Research Communications 430 1126-1131. (https://doi.org/10.1016/j.bbrc.2012.12.025)

Giordano A, Frontini A \& Cinti S 2016 Convertible visceral fat as a therapeutic target to curb obesity. Nature Reviews: Drug Discovery 15 405-424. (https://doi.org/10.1038/nrd.2016.31)

Gusarova V, Banfi S, Alexa-Braun CA, Shihanian LM, Mintah IJ, Lee JS, Xin Y, Su Q, Kamat V, Cohen JC, et al. 2017 ANGPTL8 blockade with a monoclonal antibody promotes triglyceride clearance, energy expenditure, and weight loss in mice. Endocrinology 158 1252-1259. (https://doi.org/10.1210/en.2016-1894)

Hoffmann JM, Grunberg JR, Church C, Elias I, Palsdottir V, Jansson JO, Bosch F, Hammarstedt A, Hedjazifar S \& Smith U 2017 BMP4 gene therapy in mature mice reduces BAT activation but protects from obesity by browning subcutaneous adipose tissue. Cell Reports $\mathbf{2 0}$ 1038-1049. (https://doi.org/10.1016/j.celrep.2017.07.020)

Holmes D 2017 Adipose tissue: new route to functional human beige adipocytes. Nature Reviews: Endocrinology 13 251. (https://doi. org/10.1038/nrendo.2017.34)

Hwang YC, Hayashi T, Fujimoto WY, Kahn SE, Leonetti DL, McNeely MJ \& Boyko EJ 2015 Visceral abdominal fat accumulation predicts the conversion of metabolically healthy obese subjects to an unhealthy phenotype. International Journal of Obesity 39 1365-1370. (https://doi. org/10.1038/ijo.2015.75)

Ikeda K, Maretich P \& Kajimura S 2018 The Common and distinct features of brown and beige adipocytes. Trends in Endocrinology and Metabolism 29 191-200. (https://doi.org/10.1016/j.tem.2018.01.001)

Imran KM, Yoon D \& Kim YS 2018 A pivotal role of AMPK signaling in medicarpin-mediated formation of brown and beige. BioFactors $\mathbf{4 4}$ 168-179. (https://doi.org/10.1002/biof.1392)
Izumi R, Kusakabe T, Noguchi M, Iwakura H, Tanaka T, Miyazawa T, Aotani D, Hosoda K, Kangawa K \& Nakao K 2018 CRISPR/ Cas9-mediated Angptl8 knockout suppresses plasma triglyceride concentrations and adiposity in rats. Journal of Lipid Research $\mathbf{5 9}$ 1575-1585. (https://doi.org/10.1194/jlr.M082099)

Jenkins DA, Bowden J, Robinson HA, Sattar N, Loos RJF, Rutter MK \& Sperrin M 2018 Adiposity-mortality relationships in Type 2 diabetes, coronary heart disease, and cancer subgroups in the UK Biobank, and their modification by smoking. Diabetes Care 41 1878-1886. (https:// doi.org/10.2337/dc17-2508)

Lee J, Hong SW, Park SE, Rhee EJ, Park CY, Oh KW, Park SW \& Lee WY 2015 AMP-activated protein kinase suppresses the expression of LXR/SREBP-1 signaling-induced ANGPTL8 in HepG2 cells. Molecular and Cellular Endocrinology 414 148-155. (https://doi.org/10.1016/j. mce.2015.07.031)

Lu X, Altshuler-Keylin S, Wang Q, Chen Y, Henrique Sponton C, Ikeda K, Maretich P, Yoneshiro T \& Kajimura S 2018 Mitophagy controls beige adipocyte maintenance through a Parkin-dependent and UCP1independent mechanism. Science Signaling 11 eaap8526. (https://doi. org/10.1126/scisignal.aap8526)

Mottillo EP, Desjardins EM, Crane JD, Smith BK, Green AE, Ducommun S, Henriksen TI, Rebalka IA, Razi A, Sakamoto K, et al. 2016 Lack of adipocyte AMPK exacerbates insulin resistance and hepatic steatosis through brown and beige adipose tissue function. Cell Metabolism $\mathbf{2 4}$ 118-129. (https://doi.org/10.1016/j.cmet.2016.06.006)

Qian SW, Tang Y, Li X, Liu Y, Zhang YY, Huang HY, Xue RD, Yu HY, Guo L, Gao HD, et al. 2013 BMP4-mediated brown fat-like changes in white adipose tissue alter glucose and energy homeostasis. PNAS 110 E798-E807. (https://doi.org/10.1073/pnas.1215236110)

Tseng YH, Yeh YH, Chen WJ \& Lin KH 2014 Emerging regulation and function of betatrophin. International Journal of Molecular Sciences $\mathbf{1 5}$ 23640-23657. (https://doi.org/10.3390/ijms151223640)

Veniant MM, Sivits G, Helmering J, Komorowski R, Lee J, Fan W, Moyer C \& Lloyd DJ 2015 Pharmacologic effects of FGF21 are independent of the 'browning' of white adipose tissue. Cell Metabolism 21 731-738. (https://doi.org/10.1016/j.cmet.2015.04.019)

von Loeffelholz C, Pfeiffer AFH, Lock JF, Lieske S, Docke S, Murahovschi V, Kriebel J, de Las Heras Gala T, Grallert H, Rudovich N, et al. 2017 ANGPTL8 (Betatrophin) is expressed in visceral adipose tissue and relates to human hepatic steatosis in two independent clinical collectives. Hormone and Metabolic Research 49 343-349. (https://doi.org/10.1055/s-0043-102950)

Wang C, Tong Y, Wen Y, Cai J, Guo H, Huang L, Xu M, Feng M, Chen X, Zhang J, et al. 2018 Hepatocellular carcinoma-associated protein TD26 interacts and enhances sterol regulatory elementbinding protein 1 activity to promote tumor cell proliferation and growth. Hepatology 68 1833-1850. (https://doi.org/10.1002/ hep.30030)

Zebisch K, Voigt V, Wabitsch M \& Brandsch M 2012 Protocol for effective differentiation of 3T3-L1 cells to adipocytes. Analytical Biochemistry 425 88-90. (https://doi.org/10.1016/j.ab.2012.03.005)

Zhang R 2012 Lipasin, a novel nutritionally-regulated liver-enriched factor that regulates serum triglyceride levels. Biochemical and Biophysical Research Communications 424 786-792. (https://doi. org/10.1016/j.bbrc.2012.07.038)

Zhang S, Cao H, Li Y, Jing Y, Liu S, Ye C, Wang H, Yu S, Peng C, Hui L, et al. 2018 Metabolic benefits of inhibition of p38alpha in white adipose tissue in obesity. PLoS Biology 16 e2004225. (https://doi. org/10.1371/journal.pbio.2004225)

Received in final form 21 January 2020

Accepted 6 February 2020

Accepted Manuscript published online 6 February 2020 https://joe.bioscientifica.com https://doi.org/10.1530/JOE-19-0447 (c) 2020 Society for Endocrinology Published by Bioscientifica Ltd. 